\title{
Helicobacter pylori infection is correlated with the incidence of erosive oral lichen planus and the alteration of the oral microbiome composition
}

Shutong $\mathrm{Li}^{1,2+}$, Yangheng Zhang ${ }^{3 \dagger}$, Zongcheng Yang ${ }^{4}$, Jingyuan $\mathrm{Li}^{2}, \mathrm{Ya} \mathrm{Li}^{1}, \mathrm{H}$ Hanjie $\mathrm{Li}^{5}$, Wenjuan $\mathrm{Li}^{1}$, Jihui Jia ${ }^{1}$, Shaohua $\mathrm{Ge}^{2^{*}}$ and Yundong Sun ${ }^{1 *}$

\begin{abstract}
Background: Oral lichen planus (OLP), a common clinical oral disease, is associated with an increased risk of malignant transformation. The mechanism underlying the pathogenesis of OLP is unknown. Oral dysbacteriosis is reported to be one of the aetiological factors of OLP. Although Helicobacter pylori infection is associated with various oral diseases, the correlation between $\mathrm{H}$. pylori infection and OLP is unclear. This study aimed to investigate the effect of $\mathrm{H}$. pylori infection on OLP pathogenesis and oral microbiome composition in the Chinese population, which has a high incidence of $H$. pylori infection.
\end{abstract}

Result: In this study, saliva samples of 30 patients with OLP (OLP group) and 21 negative controls (NC group) were collected. H. pylori infection was detected using the carbon-13-labeled urea breath test (UBT). The saliva samples were divided into the following four groups based on the H. pylori status: H. pylori-positive OLP (OLP+), H. pyloripositive NC (NC+), H. pylori-negative OLP (OLP-), and H. pylori-negative NC (NC-). Oral microbiome compositions were significantly different between the OLP and NC groups and between the OLP- and OLP+ groups. Compared with those in the OLP - group, those in the OLP+ group had a higher incidence of erosive OLP and higher levels of salivary cytokines. In contrast, the oral microbiome composition and cytokine levels were not significantly different between the NC- and NC+ groups.

Conclusions: This is the first report to demonstrate that $H$. pylori infection is significantly correlated with the pathogenesis of erosive OLP.

Keywords: Helicobacter pylori, Oral lichen planus, Saliva, Microbiome, Inflammatory factor

\footnotetext{
* Correspondence: shaohuage@sdu.edu.cn; syd@sdu.edu.cn

†'Shutong Li and Yangheng Zhang contributed equally to this work.

${ }^{2}$ Department of Periodontology, School and Hospital of Stomatology,

Cheeloo College of Medicine, Shandong University \& Shandong Key

Laboratory of Oral Tissue Regeneration \& Shandong Engineering Laboratory

for Dental Materials and Oral Tissue Regeneration, Jinan 250012, Shandong,

China

'Key Laboratory for Experimental Teratology of Ministry of Education and Department of Microbiology, School of Basic Medical Science, Cheeloo College of Medicine, Shandong University, Jinan 250012, Shandong, China

Full list of author information is available at the end of the article
}

(c) The Author(s). 2021 Open Access This article is licensed under a Creative Commons Attribution 4.0 International License, which permits use, sharing, adaptation, distribution and reproduction in any medium or format, as long as you give appropriate credit to the original author(s) and the source, provide a link to the Creative Commons licence, and indicate if changes were made. The images or other third party material in this article are included in the article's Creative Commons licence, unless indicated otherwise in a credit line to the material. If material is not included in the article's Creative Commons licence and your intended use is not permitted by statutory regulation or exceeds the permitted use, you will need to obtain permission directly from the copyright holder. To view a copy of this licence, visit http://creativecommons.org/licenses/by/4.0/ The Creative Commons Public Domain Dedication waiver (http://creativecommons.org/publicdomain/zero/1.0/) applies to the data made available in this article, unless otherwise stated in a credit line to the data. 


\section{Background}

Infection with Helicobacter pylori (H. pylori), which is classified as a primary carcinogen by the World Health Organization (WHO), can lead to the development of chronic gastritis, gastric ulcer, and gastric cancer $[1,2]$. The human oral cavity is a temporary extragastric $H$. pylori reservoir [3]. H. pylori has been detected in dental plaques and saliva using various molecular techniques $[4,5]$. Additionally, $H$. pylori infection has been associated with oral diseases, such as periodontitis and recurrent oral ulcers $[5,6]$.

The prevalence of oral lichen planus (OLP), a common chronic inflammatory oral mucosal disease, is $0.5-2.2 \%$ in the general adult population [7]. The two main subtypes of OLP are reticular OLP and erosive OLP. Reticular OLP is characterized by protruding white lacy lesions and papules $[7,8]$, while the erosive OLP is characterized by erythema, erosion, and ulcerative lesions [7, 8]. OLP can cause variable symptoms with different degrees of discomfort, including a prolonged burning sensation, bleeding, tingling, and abnormal taste. Previous studies have demonstrated that OLP, which is defined as a precancerous condition by the WHO, is associated with an increased risk of malignant transformation [9].

Various factors, such as immunological, psychological, infectious, and genetic factors, are reported to be involved in the pathogenesis of OLP $[8,10,11]$. However, the correlation between $H$. pylori infection and OLP has not been elucidated. $H$. pylori has been detected in the periodontal pockets of patients with OLP. Additionally, the presence of $H$. pylori in the oral cavity is associated with leucoplakia and OLP-associated oral lesions [6]. One study detected $H$. pylori nucleic acids in erosive OLP lesions and suggested a possible connection between $H$. pylori and erosive OLP, but they did not perform a statistical analysis [12]. Nevertheless, recent studies have reported that $H$. pylori infection was not detected in the mucosal biopsies of patients with OLP, indicating no correlation between OLP and $H$. pylori infection [13]. Hence, previous studies evaluating the correlation between OLP and H. pylori reported contradictory findings. Additionally, most of these studies involved Western populations. In Chinese populations, the average $H$. pylori infection rate is high (more than 50\%) [14]. However, the correlation between $H$. pylori and OLP has not been examined in the Chinese population.

Recent studies have demonstrated that the oral microbiome is involved in the development of oral diseases [15]. Oral dysbacteriosis is reported to be one of the aetiological factors of OLP [16]. Indeed, H. pylori infection alters the microbiome composition and decreases microbial diversity $[17,18]$. One study showed that the eradication of $H$. pylori resulted in changes in the gastric microbiome, including an increase in bacterial diversity
[18]. Other studies also found that $H$. pylori infection might be correlated with gut bacteria dysbiosis [19]. Similar results were reported in children with $H$. pyloriinduced gastritis [20]. However, the impact of H.pylori infection on the oral microbiome still needs further research.

In this study, we aimed to explore the correlation between $H$. pylori infection and OLP. Thus, saliva samples collected from normal control volunteers and patients with OLP were grouped based on $H$. pylori infection status. The clinical subtypes of OLP in $H$. pylori-positive and $H$. pylori-negative patients were determined. The findings of this study indicated that $H$. pylori infection was correlated with an increased incidence of erosive OLP. However, the underlying mechanism was not elucidated. Therefore, the oral microbiome composition of OLP patients with and without $H$. pylori infection was examined to evaluate the effect of $H$. pylori infection on the oral microbiome composition.

\section{Results \\ Patients with OLP had an increased prevalence of $H$. pylori infection}

Saliva samples of 30 patients with OLP (OLP group) and 21 normal control volunteers (NC group) were collected to analyse the correlations between $H$. pylori infection status and OLP clinical subtypes. Age and sex were not significantly different between the OLP and NC groups (Table 1).

H. pylori infection in the OLP and NC groups was detected using the 13C-UBT. The prevalence of $H$. pylori infection in the OLP group (70\%) was markedly higher than that in the NC group (50\%) (Table 1).

Erosive OLP was characterized by a red erosive mucosal surface (Fig. S1 in Additional file). Lesion severity and risk of malignant transformation were higher in those with erosive OLP than in those with reticular OLP [9]. In the OLP group, the incidence of erosive OLP in H. pylori-positive patients $(61.9 \%)$ was significantly (chisquare test) higher than that in $H$. pylori-negative patients $(11.1 \%)(p=0.0041)$. Additionally, $H$. pylori infection was correlated with OLP subtypes (Table 2 ).

\section{Patients with OLP exhibited an altered oral microbiome composition}

Previous studies demonstrated that the oral microbiome composition in patients with OLP was different than that in normal control individuals [21-23]. This study demonstrated that the $\alpha$ diversity of the oral microbiome in the OLP group was significantly higher than that in the NC group (Fig. 1a and b). Principal coordinate analysis (PCoA) revealed that the $\beta$ diversity of the oral microbiome in the OLP group was significantly different than that in the NC group (Fig. 1c). 
Table 1 Demographic and clinical parameters of the saliva study samples found no statistically significant differences in age and gender of each group

\begin{tabular}{|c|c|c|c|c|c|}
\hline Characteristics & $\begin{array}{l}\text { NC- } \\
(n=10)\end{array}$ & $\begin{array}{l}\mathrm{NC}+ \\
(n=11)\end{array}$ & $\begin{array}{l}\text { OLP- } \\
(n=9)\end{array}$ & $\begin{array}{l}\text { OLP+ } \\
(n=21)\end{array}$ & $\begin{array}{l}p \\
\text { value }\end{array}$ \\
\hline Age (mean $\pm S D)$ & $46.44 \pm 3.772$ & $48.30 \pm 3.211$ & $48.30 \pm 3.211$ & $46.71 \pm 2.618$ & $0.45^{\mathrm{a}}$ \\
\hline Male/female & $7 / 3$ & $11 / 0$ & $8 / 1$ & $16 / 5$ & $0.5^{\mathrm{b}}$ \\
\hline Smoking habit; & 0 & 0 & 0 & 0 & \\
\hline Alcohol drinking; & 0 & 0 & 0 & 0 & \\
\hline Antibiotics usage; & 0 & 0 & 0 & 0 & \\
\hline Systemic disease; & 0 & 0 & 0 & 0 & \\
\hline
\end{tabular}

${ }^{\mathrm{a} A N O V A},{ }^{\mathrm{b}}$ Fisher's test

Oral microbiome compositions were significantly different between the OLP and NC groups at the phylum (Fig. 1d) and genus levels (Fig. 1e). The dominant oral phyla were Firmicutes, Proteobacteria, and Bacteroidetes (Fig. 1d). Compared with the NC group, the OLP group had a significantly decreased abundance of Firmicutes and a significantly increased abundance of Bacteroidetes (Fig. 1d). At the genus level, compared with the NC group, the OLP group exhibited a decreased abundance of Streptococcus and increased abundances of Neisseria, Prevotella, and Prevotella7 (Fig. 1e).

The bacterial genera with average relative abundances higher than $1 \%$ in the oral microbiomes in the OLP and NC groups are listed in Fig. 2a. A volcano plot was used to visalize the differences in the bacterial composition at the genus level between the OLP and NC groups (Fig. $2 b)$. Bacteria with relative abundances greater than $1 \%$ and significant differences in relative abundances were screened out (Fig. 2b). Compared with the NC group, the OLP group exhibited decreased relative abundances of Streptococcus and Rothia and increased relative abundances of Alloprevotella, Prevotella, Fusobacterium, and Porphyromonas (Fig. 2b and c).

\section{H. pylori infection alters the salivary microbiome composition in patients with OLP}

To analyse the effect of $H$. pylori infection on the salivary microbiome composition in the OLP and NC groups, the saliva samples were divided into the following four groups based on $H$. pylori infection status: OLP+ $(n=$ $21)$; OLP- $(n=9)$; NC+ $(n=10)$; NC- $(n=11)$.

Table 2 Chi-sauared test showed statistically significant association of infection of $\mathrm{H}$. pylori and the alteration of OLP subtype $(p<0.005)$

\begin{tabular}{llcl}
\hline OLP subtype (\%) & $\begin{array}{l}\text { OLP- } \\
(\boldsymbol{n}=\mathbf{9})\end{array}$ & $\begin{array}{l}\text { OLP+ } \\
(\boldsymbol{n}=\mathbf{2 1})\end{array}$ & $\begin{array}{l}\boldsymbol{p} \\
\text { value }\end{array}$ \\
\hline Reticular OLP $(n=16)$ & $8(88.9)$ & $8(38.1)$ & $0.0041^{\mathrm{a}}$ \\
Erosive OLP $(n=14)$ & $1(11.1)$ & $13(61.9)$ & \\
\hline
\end{tabular}

${ }^{a}$ Chi-square test
The $\alpha$ diversity of the salivary microbiome in the OLP+ group was significantly higher than that in the OLP- group (Fig. 3a, b, c, d and e). In contrast, the $\alpha$ diversity was not significantly different between the $\mathrm{NC}+$ and NC- groups (Fig. 3a, b, c, d and e). PCoA revealed that the $\beta$ diversity of the salivary microbiome was significantly different between the OLP+ and OLP- groups. However, the $\beta$ diversity of the salivary microbiome was not significantly different between the $\mathrm{NC}+$ and $\mathrm{NC}-$ groups (Fig. $3 \mathrm{f}$ and g). Additionally, the bacterial compositions at the genus and phylum levels were not significantly different between the $\mathrm{NC}+$ and $\mathrm{NC}-$ groups (Fig. S2 in Additional file).

The salivary microbiome compositions were significantly different between the OLP+ and OLP- groups at the phylum and genus levels (Fig. 4a and b). The predominant bacterial phyla were Proteobacteria, Firmicutes, and Bacteroidetes (Fig. 4a), and the relative abundance of Bacteroidetes was significantly higher in the OLP+ group than in the OLP- group. At the genus level, the relative abundance of Alloprevotella in the OLP+ group was significantly higher than that in the OLP- group (Fig. 4b).

The bacteria of the salivary microbiome in the OLP+ and OLP- groups with an average relative abundances higher than 1\% are listed in Fig. 4c. A volcano plot was constructed to visualize the differences in the bacterial composition between the OLP+ and OLP- groups at the genus level (Fig. 4d). Bacteria with a relative abundance higher than $1 \%$ and significant differences in the relative abundance were screened out (Fig. 4d). Compared with the OLP - group, the OLP+ group exhibited significantly increased relative abundances of Alloprevotella and Haemophilus and a significantly decreased relative abundance of Actinomyces (Fig. 4d and e).

\section{Comparative analysis of salivary inflammatory factors}

H. pylori infection can induce the gastric mucosa to secrete inflammatory factors, such as IL-6, IL-8, and IL-17 [24]. Previous studies have reported the dysregulated expression of various inflammatory factors, such as IL-6, 

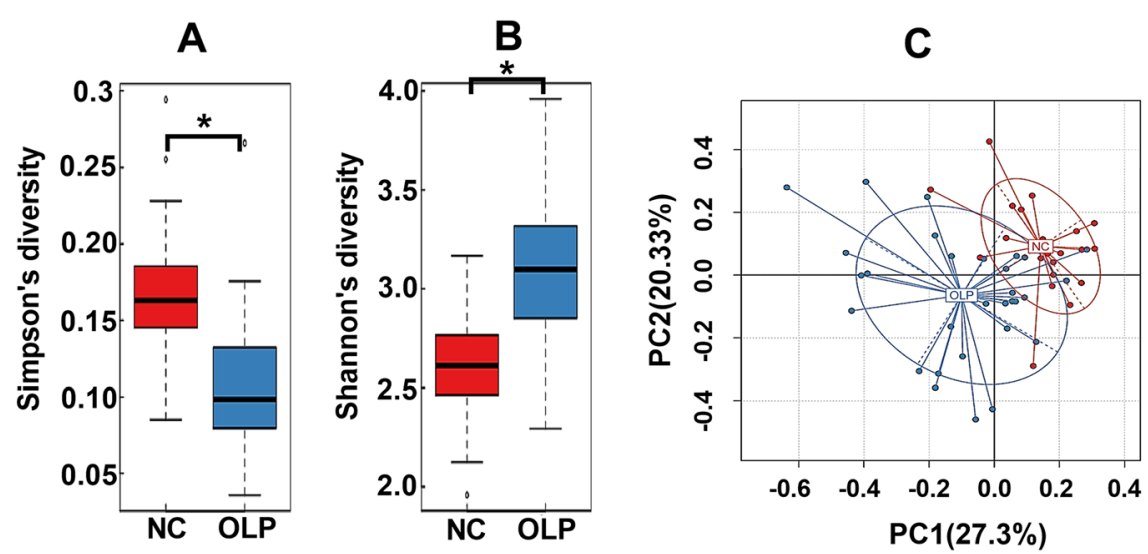

D

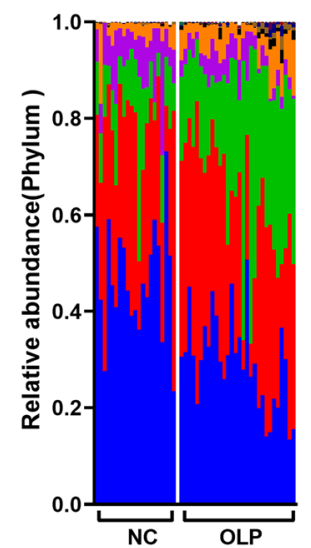

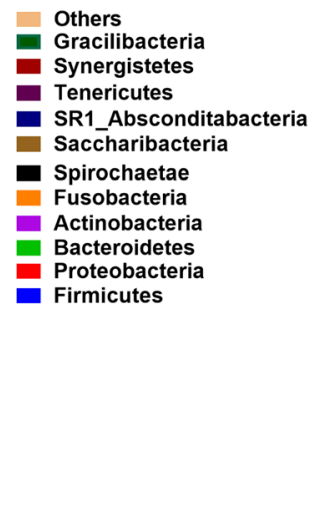



Fig. 1 Comparison of the salivary microbiome profiles between the NC $(n=21)$ and OLP groups $(n=30)$. a Simpson's diversity index of salivary microbiome in the NC and OLP groups $(p=0.00003)$. $\mathbf{b}$ Shannon's diversity index of salivary microbiome in the NC and OLP groups $(p=0.0002)$. $\mathbf{c}$ Principal coordinate analysis (PCOA) plots of unweighted UniFrac distances of oral microbiota in the NC and OLP groups. Comparative analysis of the oral microbiome composition at the phylum (d) and genus (e) levels between the NC and OLP groups. ${ }^{*} p<0.05$

IL-8, IL-17, and TNF- $\alpha$ in patients with OLP [25]. In this study, the salivary levels of IL-6, IL-8, and IL-17 in the OLP and NC groups were analysed using ELISA. The salivary levels of IL-6, IL-8, and IL-17 in the OLP group were significantly higher than those in the $\mathrm{NC}$ group (Fig. 5a).

Next, the effect of $H$. pylori infection on the salivary levels of inflammatory factors in the OLP and NC groups was evaluated. Additionally, the salivary levels of IL-6, IL-8, and IL-17 were comparatively analysed between the following groups: the OLP+ and OLP- groups and the $\mathrm{NC}+$ and $\mathrm{NC}-$ groups. Compared with those in the OLP- group, the salivary levels of IL-6, IL-8, and IL17 were significantly increased in the OLP+ group (Fig. 5b). However, the salivary levels of IL-6, IL-8, and IL-17 were not significantly different between the $\mathrm{NC}+$ and $\mathrm{NC}$ - groups (Fig. 5c).

Next, the correlations between key bacterial genera and inflammatory factors (IL-6, IL-8, and IL-17) were analysed by constructing a heat map of Spearman's rank correlation coefficients (Fig. 6a and b). In the OLP and NC groups, the abundances of the Alloprevotella, Porphyromonas, Fusobacterium, and Prevotella genera were positively correlated with IL-6 and IL-17, while the abundances of the Prevotella and Fusobacterium genera were positively correlated with IL-8. Furthermore, the abundances of the Streptococcus and Rothia genera were negatively correlated with IL-7, IL-6, and IL-8 (Fig. 6a). In the OLP+ and OLP- groups, the abundances of the Alloprevotella and Haemophilus genera were significantly and positively correlated with IL-17, while those of the Actinomyces genus were negatively correlated with IL-7, IL-6, and IL-8 (Fig. 6b).

\section{Correlation of salivary microbiome function with key bacterial genera}

PICRUSt was used to predict the metagenome functional content based on 16S rRNA gene sequencing and Kyoto 
A

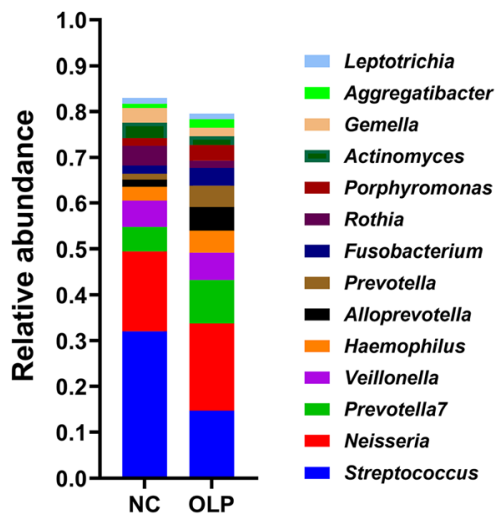

B

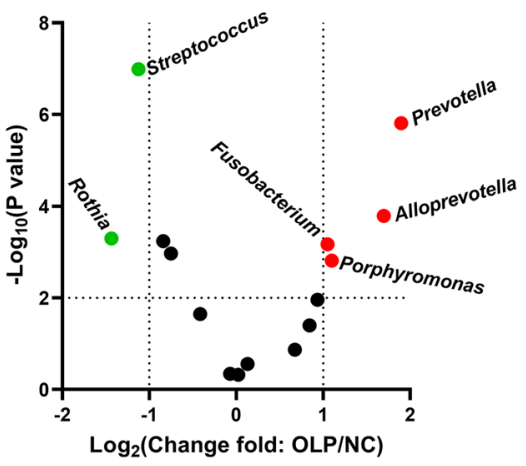

C

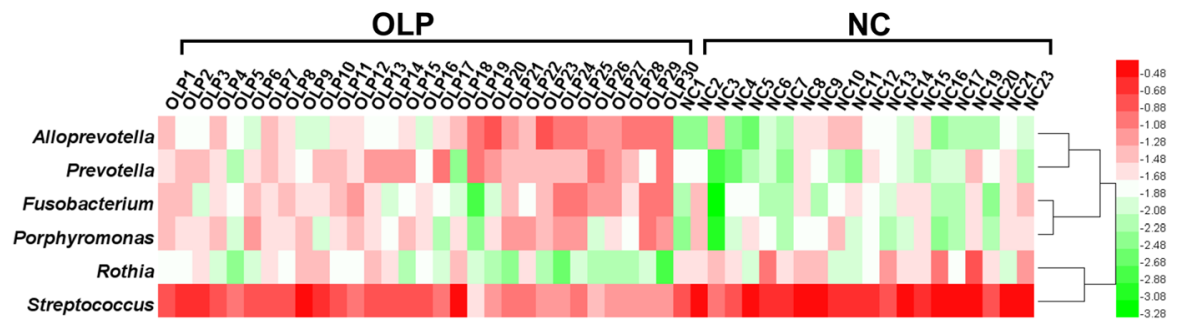

Fig. 2 Comparison of the salivary microbiome composition at the genus level between the NC $(n=21)$ and OLP groups $(n=30)$. a Comparative analysis of the salivary microbiome composition between the NC and OLP groups (average relative abundance > $1 \%$ in the two groups). $\mathbf{b}$ Volcano plot analysis of salivary microbiome composition between the NC and OLP groups. The $p$-value was calculated using the $t$-test. According to the results shown in panel (a), bacteria with a relative abundance greater than $1 \%$ were selected and marked as key bacteria. $\mathbf{c}$ Heat map showing the relative abundances of the key bacteria for sampled individuals of the community subgroups

Encyclopedia of Genes and Genomes (KEGG) pathway analysis (Fig. 7). Compared with those in the NC group, the expression levels of genes involved in various metabolic pathways, such as histidine metabolism, phenylalanine metabolism, novobiocin biosynthesis, lipopolysaccharide (LPS) biosynthesis, LPS biosynthesis proteins, biotin metabolism, ubiquinone, and other terpenoid-quinone biosynthesis, in the OLP group were upregulated, while those of genes involved in galactose metabolism, the phosphotransferase system, and protein kinase were downregulated (Fig. $7 \mathrm{a}$ and $\mathrm{b}$ ).

Furthermore, the levels of LPS biosynthesis proteins and LPS biosynthesis in the OLP+ group were higher than those in the OLP- group (Fig. 7c and d).

LPS can induce an inflammatory reaction [26]. PICRUSt analysis predicted that the microbial metabolic pathways involved in the pathogenesis of OLP were LPS biosynthesis proteins and LPS biosynthesis. The correlations between key bacterial genera and these two metabolic pathways were examined by constructing a heat map of Spearman's rank correlation coefficients (Fig. 8a and b). In the OLP and NC groups, the relative abundances of the Alloprevotella, Porphyromonas, Fusobacterium, and Prevotella genera were positively correlated, while those of Rothia were negatively correlated with the levels of LPS biosynthesis proteins and LPS biosynthesis (Fig. 8a). In the OLP+ and OLP- groups, the abundances of the Alloprevotella and Haemophilus genera were positively correlated, while those of Actinomyces were negatively correlated with the levels of LPS biosynthesis proteins and LPS biosynthesis (Fig. 8b).

\section{Discussion}

This study aimed to analyse the correlation between $H$. pylori infection and the pathogenesis of OLP. The analysis of clinical data indicated that $H$. pylori infection was correlated with an increased incidence of erosive OLP. Patients with OLP exhibited distinct salivary microbiome compositions and increased salivary levels of inflammatory factors. H. pylori infection was correlated with an altered composition and altered function of the salivary microbiome, as well as increased salivary levels of inflammatory factors in patients with OLP but not in patients without OLP.

The pathogenesis of OLP, a common clinical oral mucosal disease, is unknown $[7,8]$. The proposed causes of OLP include genetic predisposition, cell-mediated hypersensitivity reactions, autoimmune response, stress and 


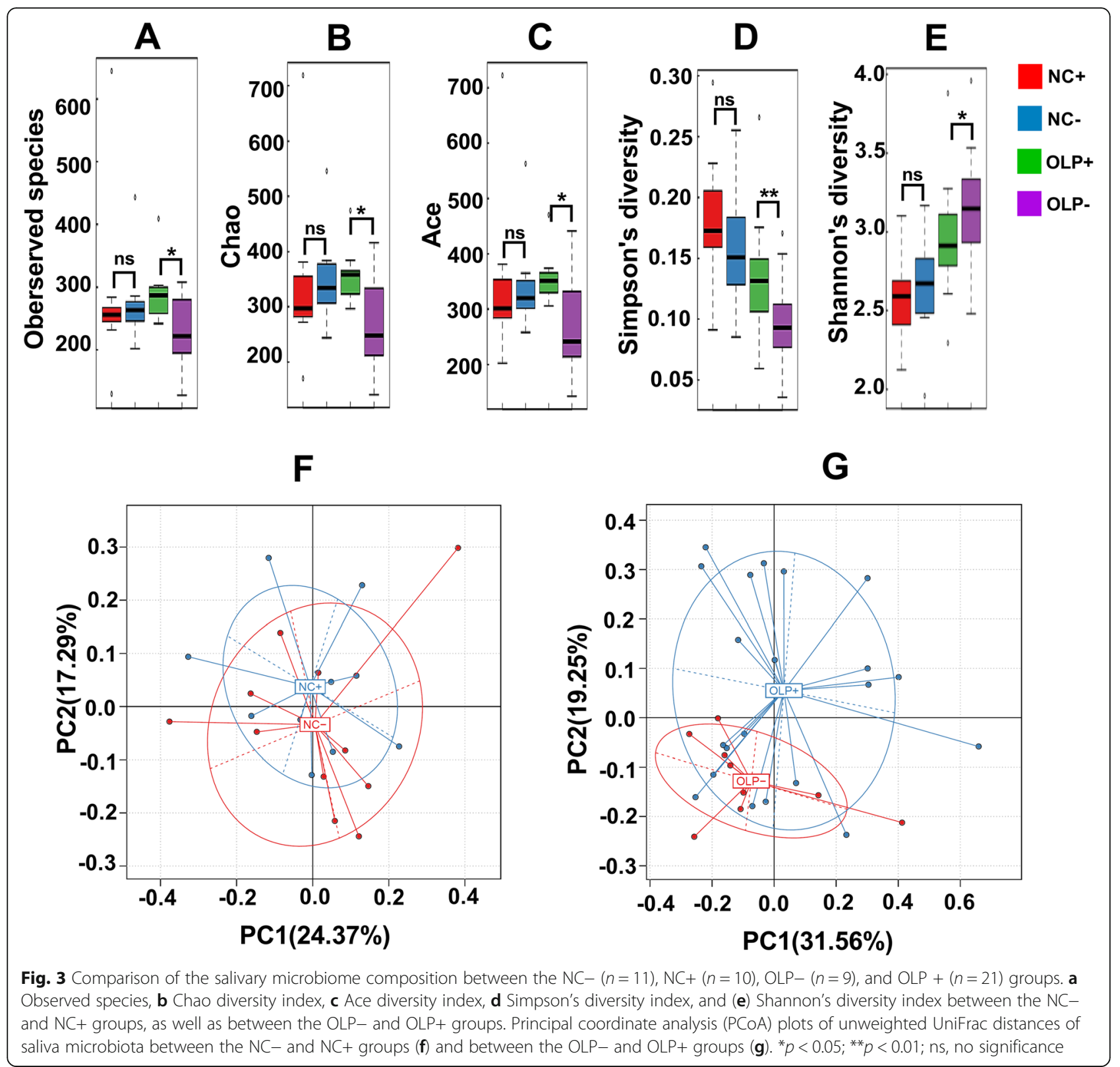

infections [8]. Among them, unbalanced cellularmediated immunity is considered the key pathogenesis of OLP, in which the increased production of $\mathrm{T}$ helper 1 (Th1) cytokines is a crucial and early event [7]. Proinflammatory macrophages may exacerbate OLP performance by producing cytokines, such as IL-6, IL-8, and IL17 [27]. Moreover, oral dysbacteriosis has recently been reported to be one of the aetiological factors of OLP [16]. Studies reported that the oral microbiome of patients with OLP was characterised by increased microbial diversity, decreased Streptococcus abundance, and increased Bacteroides abundance [28-30].

This study, for the first time, demonstrated that $H$. pylori infection is significantly correlated with the pathogenesis of erosive OLP. One of the main pathogenic mechanisms of $H$. pylori infection is damage to host by inducing local and systemic inflammation, with which Th1 and Th17 cells are associated [2, 31, 32]. IL6, IL-17 and IL-8 are reported to play an important role in $H$. pylori infection-induced inflammation [31, 33-36]. H. pylori infection-induced inflammatory cytokines in the stomach can enter the bloodstream and reach the oral cavity to modulate the immune microenvironment in the oral cavity and aggravate the inflammatory response [37]. In this study, we found that the concentrations of IL-6, IL-8, and IL-17, which are all involved in the pathogenesis of erosive OLP [38], were significantly higher in OLP patients, especially OLP patients with $H$. 


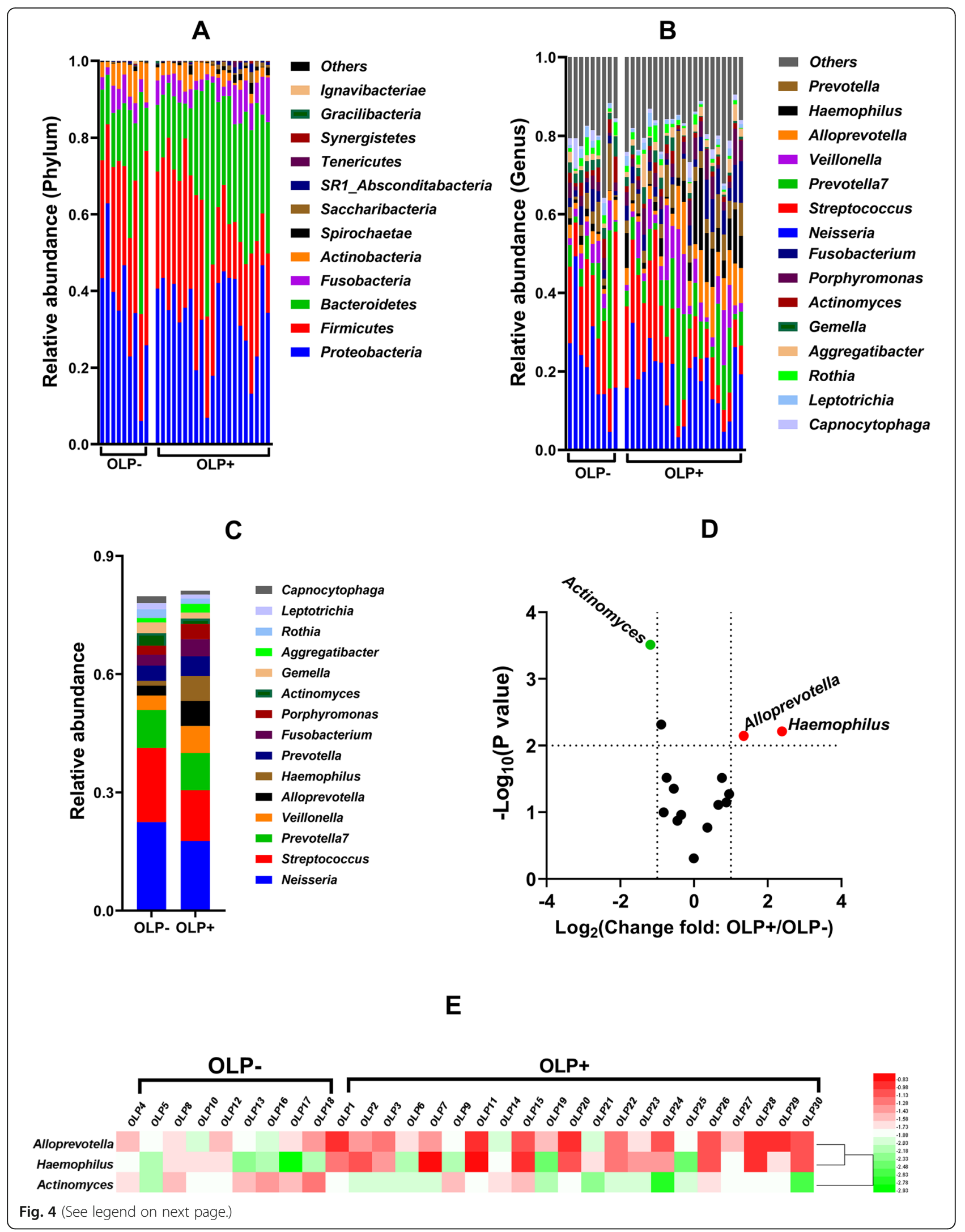


(See figure on previous page.)

Fig. 4 Comparative analysis of the salivary microbiome composition between the OLP- $(n=9)$ and OLP+ $(n=21)$ groups. a Comparative analysis of the salivary microbiome composition at the phylum and (b) the genus levels between the OLP- and OLP + groups. c Comparative analysis of the salivary microbiome composition between the OLP- and OLP+ groups (average relative abundance $>1 \%$ in the two groups). $\mathbf{d}$ Volcano plot analysis of salivary microbiome composition between the OLP- and OLP+ groups. The $p$-value was calculated using the $t$-test. According to the results shown in panel $(\mathbf{c})$, bacteria with a relative abundance greater than $1 \%$ were selected and marked as key bacteria. e Heat map showing the relative abundance of the key bacteria for sampled individuals of the community subgroups

pylori infection; this may contribute to the pathogenicity of erosive OLP.

The microbiome and the immune microenvironment influence each other [39], and changes in the local immune microenvironment can change the composition of the microbiome [40]. In patients with OLP, H. pylori infection may exacerbate the pathological condition of the oral immune microenvironment and disrupt the homeostasis of the salivary microbiome through interactions with different members of the microbial community [41]. This study demonstrated for the first time that $H$. pylori infection is significantly correlated with alterations in the microbiome in OLP patients. Consistent with the findings of previous studies, this study demonstrated that the salivary microbiome composition in patients with OLP was significantly different than that in participants without OLP [21-23]. In this study, OLP patients with $H$. pylori infection exhibited a decreased relative abundance of Actinomyces in the salivary microbiome. Actinomyces spp., which are core microorganisms in the oral cavity [42], are essential for maintaining the balance of bacterial flora. The decreased abundance of dominant bacteria will lead to the dysregulation of microbiome composition and promote the colonization of other bacteria [43]. In this study, the relative abundances of Alloprevotella and Haemophilus in the salivary microbiome were significantly higher in the OLP+ group than in the OLP- group. Haemophilus spp., gram-negative bacilli, are associated with various opportunistic infections [44-46]. Additionally, Haemophilus spp. can activate the macrophages and promote the secretion of proinflammatory cytokines, such as IL-6 and IL-8 [47]. Furthermore, Haemophilus spp. infection can induce Th17 cell differentiation and IL-17 secretion and accelerate the recruitment of neutrophils [48].

Next, we analysed the changes in oral microbiome functions caused by $H$. pylori infection. Salivary microorganisms associated with LPS synthesis metabolic pathways were significantly upregulated in OLP patients with $H$. pylori infection and were positively correlated with the relative abundances of Haemophilus and Alloprevotella. LPS, which exerts immunostimulatory and proinflammatory effects, can promote local inflammatory responses in tissues and upregulate the expression of inflammatory factors [26]. In epithelial cells, LPS binds to TLR4 to promote the secretion of proinflammatory cytokines and chemokines, such as IL-6 and IL-8 [49].
Additionally, LPS can stimulate the secretion of Th17related cytokines, such as IL-17, in immune cells [50]. Recent studies have demonstrated that LPS inhibits vitamin D receptors in oral keratinocytes, which impairs mucosal homeostasis and leads to epithelial barrier damage. Consequently, this may promote the occurrence and development of erosive OLP [51].

In the $\mathrm{NC}$ group, $H$. pylori infection did not enhance the secretion of salivary inflammatory cytokines or alter the microbial community structure. Previous studies have also reported that gastric $H$. pylori infection does not affect the oral microbiome composition [37]. There are conflicting reports on the colonization of $H$. pylori in the oral cavity. The number of $H$. pylori in the oral cavity is low [52]. A meta-analysis revealed that the number of $H$. pylori in saliva was less than that in dental plaque [53]. Chua et al. did not detect $H$. pylori sequences in oral swab samples [37]. In this study, it was difficult to detect $H$. pylori in saliva samples from subjects infected with $H$. pylori through 16S rRNA gene sequencing. In people without OLP, the amount of $H$. pylori in the oral cavity is limited and not enough to induce OLP. However, in patients with OLP, $H$. pylori might aggravate the progression of OLP. Therefore, we speculate that $H$. pylori infection, which is thought to be an aggravating factor rather than an initiating factor, may have no direct effect on the pathogenesis of OLP but may indirectly affect the systemic inflammatory environment and oral microbiome.

The reason for the increased incidence rate of erosive OLP in patients with $H$. pylori infection cannot be fully determined based on the findings of this study. This study elucidated the correlation of $H$. pylori infection with the oral microbiome composition and clinical phenotype of OLP.

\section{Conclusions}

This study elucidated the correlation of $H$. pylori infection with the oral microbiome composition and clinical phenotype of OLP. We demonstrated that H. pylori infection was significantly correlated with the pathogenesis of erosive OLP for the first time.

\section{Methods}

\section{Sample collection}

Patients with OLP (OLP group, $n=30$ ) who underwent comprehensive clinical and histopathological 
A


B
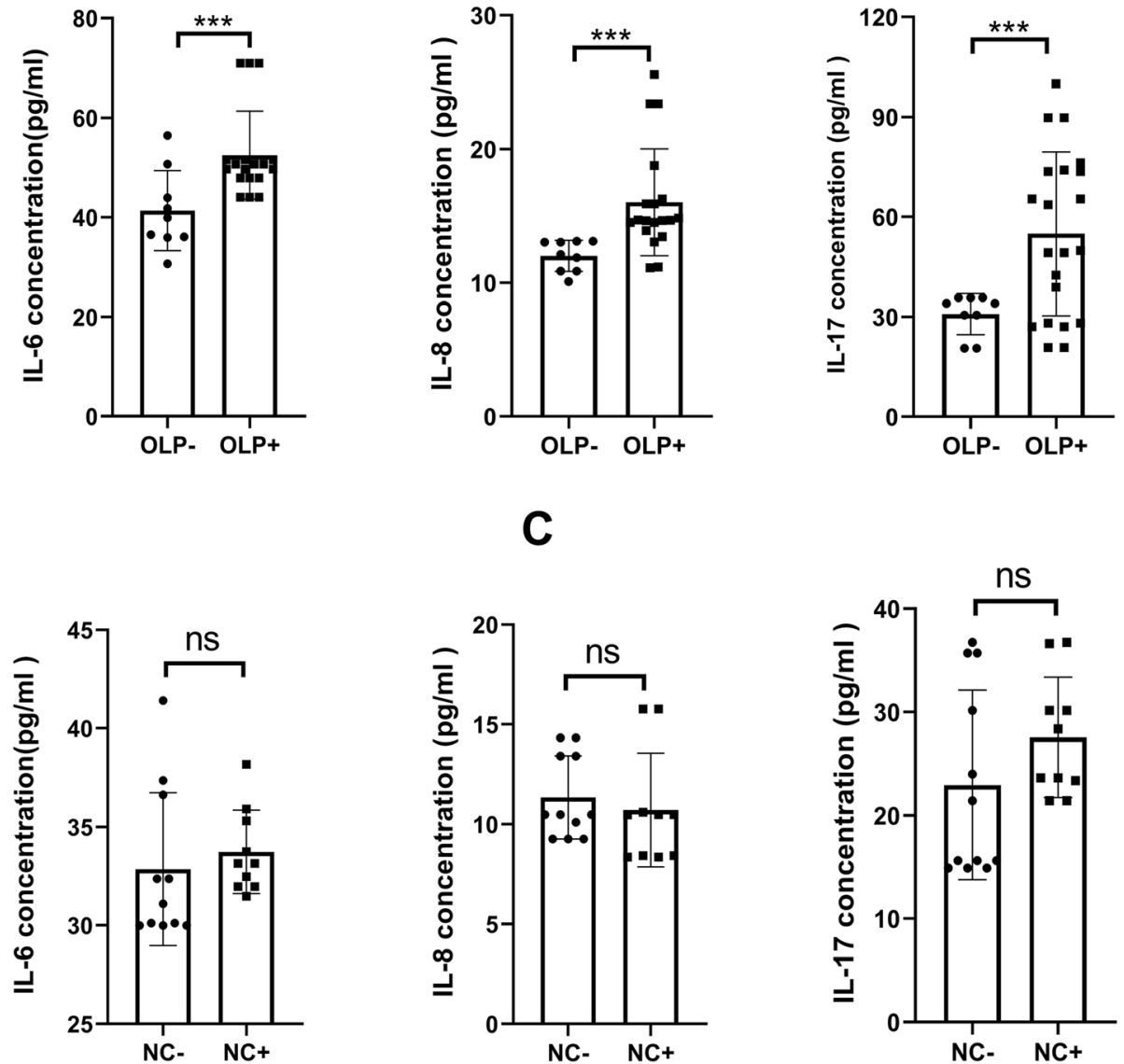

Fig. 5 The salivary levels of cytokines (IL-6, IL-8, and IL-17) were measured using ELISA. Comparative analysis of the salivary cytokine levels between the following groups: NC $(n=21)$ and OLP $(n=30)$ groups $(\mathbf{a}) ; \mathrm{OLP}-(n=9)$ and OLP+ $(n=21)$ groups $(\mathbf{b}) ; \mathrm{NC}-(n=11)$ and NC+ $(n=$ 10) groups $(\mathbf{c}) .{ }^{*} p<0.05 ;{ }^{* *} p<0.01 ;{ }^{* * *} p<0.001 ;$ ns, no significance

examinations at the Stomatology Hospital of Shandong University were randomly recruited for this study. The diagnosis of OLP was made by three experienced clinicians and pathologists. According to the clinical classification of OLP by the WHO (2005) [54], the clinical diagnostic criteria are as follows: (1) the presence of white reticular lesions, white papules or plaque-type lesions, with gray-white lines radiating from the papules; 
A



B

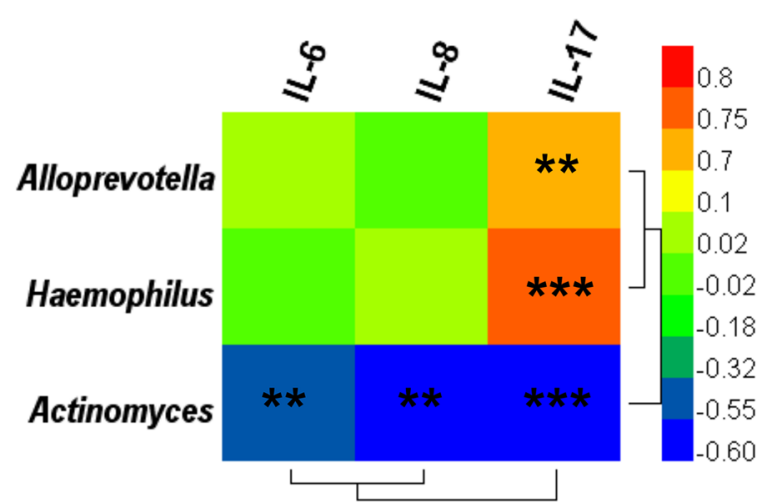

Fig. 6 Correlation of cytokine production with the relative abundance of salivary key bacteria at the genus level. a NC $(n=21)$ and OLP $(n=30)$ groups; b OLP- $(n=9)$ and OLP+ $(n=21)$ groups. The color of cells represents the correlation coefficient $(R)$ values. Cells marked with an asterisk show significance after multiple comparisons of Spearman's correlation, ${ }^{*} p<0.05 ;{ }^{* *} p<0.01 ;{ }^{* * *} p<0.001$

(2) presence of a lace-like network of slightly raised gray-white lines; and (3) presence of atrophic lesions with or without erosion and bullae. The histopathologic criteria were as follows: (1) variable epithelial thickness, with saw-tooth rete ridges sometimes observed; (2) the presence of Civatte bodies in the basal layer or superficial lamina propria; (3) the presence of a well-defined band-like zone of lymphocyte infiltration that is confined to the superficial part of the connective tissue; and (4) signs of "liquefaction degeneration" in the basal cell layer. OLP was divided into the following two subtypes: reticular OLP, characterized by white papular lesions, white reticular lesions, or white plaques; and erosive OLP, characterized by erythema and erosive lesions. According to the results of follow-up clinical examinations, agematched and sex-matched normal control volunteers were recruited (NC group, $n=21$ ). All participants' ages ranged from 18 to 60 years old. The evaluation of sample size was based on ensuring sufficient statistical power to detect whether $H$. pylori infection was related to the OLP subtype as the main result. Considering a significance level of $5 \%$ and a power of $80 \%$, according to our calculation, it was determined to be necessary to have 12 individuals for reticular OLP and 12 individuals for erosive OLP respectively. Based on previous research, we identified 51 study subjects $[23,55,56]$. After that, all the study subjects signed informed consent forms. The study subjects did not have a history of periodontitis, dental caries, systemic diseases, smoking, or alcohol consumption. Additionally, the subjects had not undergone antibiotic therapy or received OLP treatment before sample collection. This study was approved by the Medical Ethics Committee of the School of Stomatology of Shandong University (Protocol Number: 20161001).
The saliva samples were collected following the guidelines of the Manual of Procedures for the Human Microbiome Project (http://hmpdacc.org/resources/tools_ protocols.php). In brief, unstimulated saliva samples were collected from each patient between 8:00 am and 11:00 am in a sterile DNase/RNase-free conical tube. The study subjects did not consume alcohol or food for at least $2 \mathrm{~h}$ before sampling. The samples were transported to the laboratory and stored at $-80{ }^{\circ} \mathrm{C}$ until analysis.

H. pylori was detected in the OLP and NC groups using a 13C-UBT kit (Headway, Shenzhen, China). According to a previous study, the UBT technique is convenient to use, and it has a sensitivity and specificity above 90\% [57]. Based on the H. pylori infection status, the samples were divided into the following four groups: $H$. pylori-positive $\mathrm{NC}(\mathrm{NC}+, n=10), H$. pylori-negative NC (NC-, $n=11), H$. pylori-positive OLP (OLP+, $n=$ 21 ), and $H$. pylori-negative OLP (OLP-, $n=9$ ) groups.

\section{Enzyme-linked immunosorbent assay (ELISA)}

The saliva samples $(5 \mathrm{~mL})$ were centrifuged at $4{ }^{\circ} \mathrm{C}$ and $3500 \mathrm{~g}$ for $20 \mathrm{~min}$. The supernatant was stored in a DNase/RNase-free EP tube at $-80{ }^{\circ} \mathrm{C}$ until further use. The concentrations of IL-6, IL-8, and IL-17 were examined using ELISA kits (Neobioscience Technology Co., Ltd., Shenzhen, China) following the manufacturer's instructions.

\section{DNA extraction and 16S rRNA gene amplification}

Genomic DNA was isolated from the saliva samples using a QIAamp DNA micro kit (Qiagen, Valencia, CA, USA) following the manufacturer's instructions. The V3 and V4 regions (336F-806R) of the $16 \mathrm{~S}$ rRNA gene were amplified using polymerase chain reaction. 


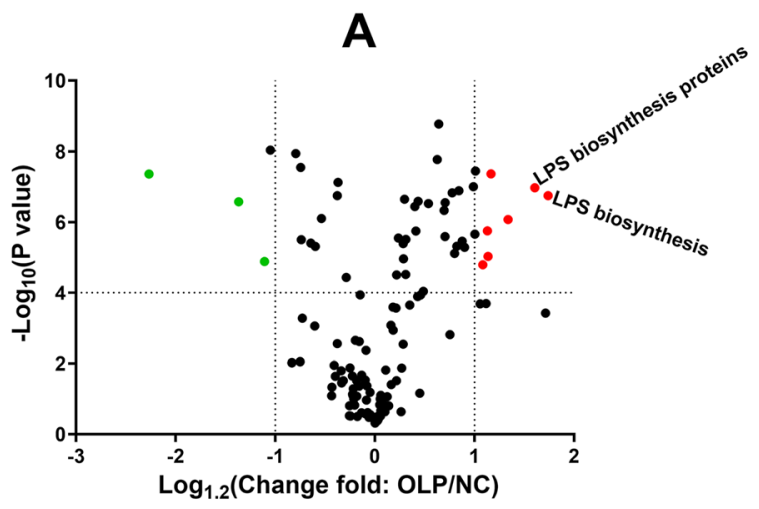

B

$\square$ NC $\square$ OLP

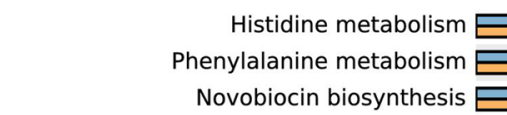

Lipopolysaccharide biosynthesis Lipopolysaccharide biosynthesis proteins Biotin metabolism

Ubiquinone and other terpenoid-quinone biosynthesis

Galactose metabolism Phosphotransferase system (PTS)

Protein kinases
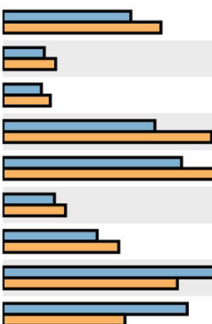

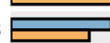

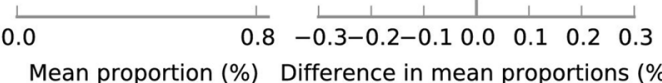

95\% confidence intervals

C



D

OLP+

Lipopolysaccharide biosynthesis proteins Lipopolysaccharide biosynthesis

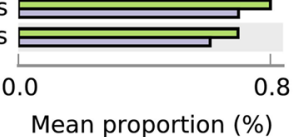



0.017 0.032
$1.18 \mathrm{e}-7$

$4.30 \mathrm{e}-6$

$2.02 \mathrm{e}-5$ के

$1.37 \mathrm{e}-6 \mathrm{U}$ $6.44 \mathrm{e}-7$ $5.37 \mathrm{e}-5$ $3.93 e-5 \frac{2}{\frac{2}{10}}$ $8.58 \mathrm{e}-5$ 구

$1.45 \mathrm{e}-7$

$3.33 \mathrm{e}-7$ 
(See figure on previous page.)

Fig. 7 Comparative analysis of the relative abundance of PICRUSt-generated functional profiles of salivary microbiota. Volcano plot analysis of altered Kyoto Encyclopedia of Genes and Genomes (KEGG) pathways between the following groups: NC $(n=21)$ and OLP $(n=30)$ groups $(\mathbf{a}) ;$ OLP- $(n=9)$ and OLP+ $(n=21)$ groups (c). KEGG pathways with an average relative abundance greater than $2 \%$ in the two groups were included. The $p$-value was calculated using the two-tailed paired $t$-test (b) and (d) based on volcano plot analysis ( $\mathbf{a}$ and $\mathbf{c}$ ). The distinct gene categories were selected according to significant differences in gene categories at level 3 ( $t$-test, $p<0.05$ ). The bar plots on the left show the mean proportion of each KEGG pathway. The dot plots on the right show the differences in mean proportions between the two indicated groups using $p$-values

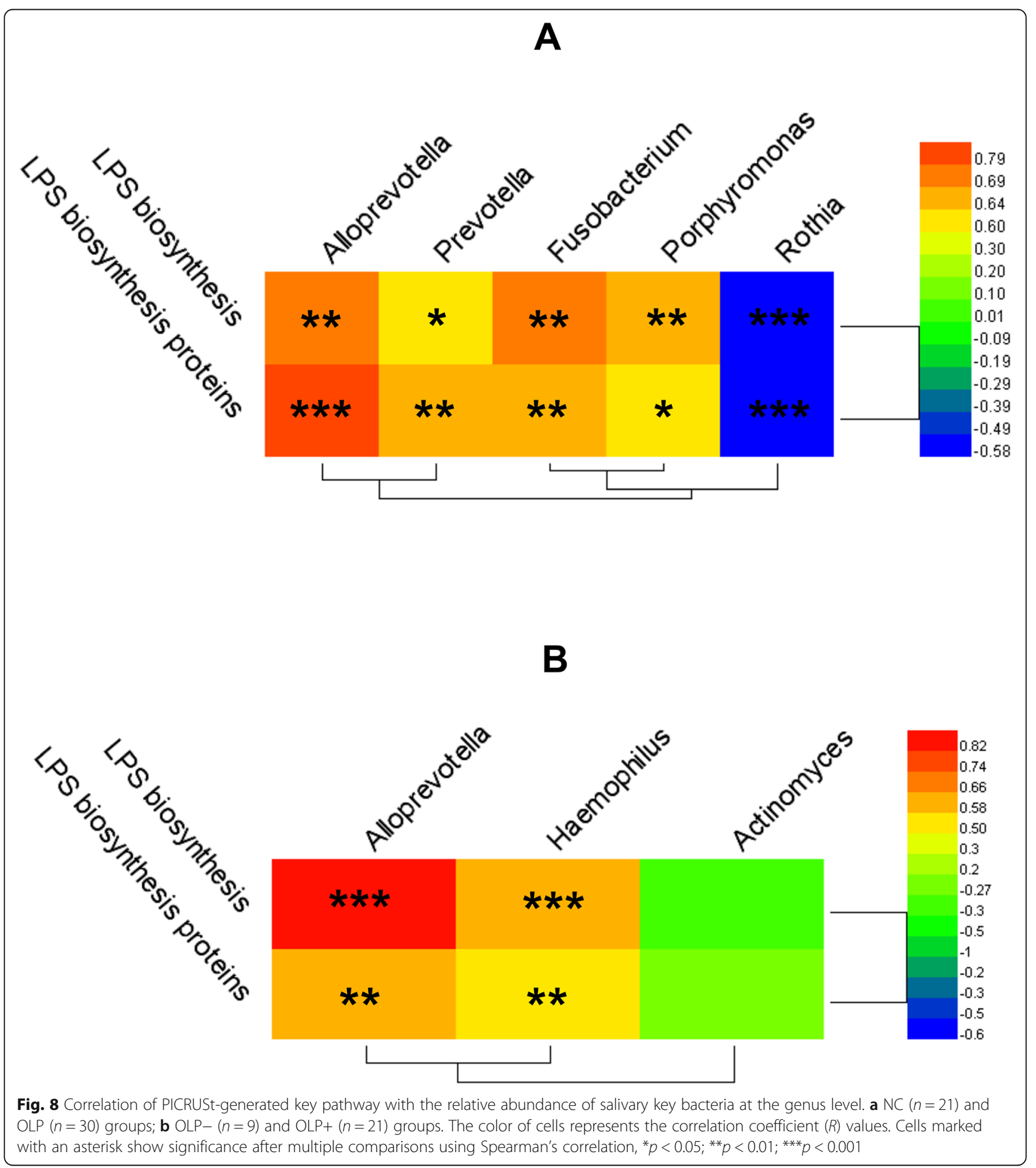




\section{Sequencing and data analysis}

High-throughput sequencing was performed at CloudSeq Biotech, Inc. (Shanghai, China). In brief, the raw sequence data were obtained from samples sequenced using an Illumina MiSeq sequencer and subjected to base calling and quality filtering. The samples were separated based on the barcodes. The adaptors were trimmed, and the low-quality reads were removed using Trimmomatic. The paired ends were merged using FLASH. The optimized reads were used for operational taxonomic unit (OTU) clustering, and OTU matrices were generated. The most abundant sequence in each OTU was selected as the representative sequence. The sequences of representative OTUs were compared with those listed in the Greengenes database and those of the samples. Taxonomic abundance matrices were generated. The determination of alpha and beta diversity, as well as statistical analysis and mapping, were performed using Mothur and the $\mathrm{R}$ environment. Microbial gene functions were predicted using PICRUSt.

\section{Statistical analysis}

All data are represented as means \pm standard deviations. The chi-square test was performed to determine the relationship between $H$. pylori infection and OLP subtype with Statistical Package for Social Science (SPSS) statistical software version 23 (SPSS Inc., Chicago IL, USA). Differences in cytokine levels between two groups were examined using the $t$-tests with GraphPad Prism version 7.01 (GraphPad Software, Inc. CA, USA). The KruskalWallis test was performed to analyse the differences in microbiome compositions between two groups with GraphPad Prism version 9 (GraphPad Software, Inc. CA, USA). The correlation between variables was examined using Spearman's correlation test with Statistical Package for Social Science (SPSS) statistical software version 23 (SPSS Inc., Chicago IL, USA). A p-value below 0.05 was considered significant. ${ }^{*} p<0.05 ;{ }^{* *} p<0.01$; ${ }^{* * *} p<$ 0.001 .

\section{Supplementary Information}

The online version contains supplementary material available at https://doi. org/10.1186/s12866-021-02188-0.

\section{Additional file 1.}

\section{Acknowledgements}

We are grateful to Ding Chao doctor from Medical School of Nanjing University for his very kind help in analyzing our data. In particular, we would like to thank Dr. Junze Liu from the Department of Computer Science at University of California, Irvine for his constant encouragement.

\section{Authors' contributions}

YS \& SG: conceptualized and supervised this study; YS, SL \& ZY: study design and original draft preparation; WL, YS, JJ \& SG: acquired funding; SL, YZ, JL, HL \& YL: analyzed the data; YS \& SL: involved in writing and editing. All authors have read and approved the manuscript.

\section{Funding}

This research was subsidized by the National Natural Science Foundation of China (Nos. 81671978, 81471991and 81772143) and the Department of Science and Technology of Shandong Province (Nos. 2018CXGC1208).

\section{Availability of data and materials}

The sequencing data generated in this study are submitted to the Sequence Read Archive of the National Center for Biotechnology Information database (accession number: SRP133987). All data supporting the findings of this study are available in the manuscript. Supplementary material can be obtained from the corresponding author upon reasonable request.

\section{Declarations}

Ethics approval and consent to participate

The experimental protocol was established, according to the ethical guidelines of the Helsinki Declaration and was approved by the Medical Ethics Committee of School of Stomatology of Shandong University (Protocol Number: 20161001). Written informed consent was obtained from individual or guardian participants.

Consent for publication

Not applicable.

\section{Competing interests}

The authors declare no conflict of interest.

\section{Author details}

${ }^{1}$ Key Laboratory for Experimental Teratology of Ministry of Education and Department of Microbiology, School of Basic Medical Science, Cheeloo College of Medicine, Shandong University, Jinan 250012, Shandong, China. ${ }^{2}$ Department of Periodontology, School and Hospital of Stomatology, Cheeloo College of Medicine, Shandong University \& Shandong Key Laboratory of Oral Tissue Regeneration \& Shandong Engineering Laboratory for Dental Materials and Oral Tissue Regeneration, Jinan 250012, Shandong, China. ${ }^{3}$ Department of Periodontology, Nanjing Stomatological Hospital, Medical School of Nanjing University, 30 Zhongyang Road, Nanjing 210008 , China. ${ }^{4}$ Department of Implantology, School and Hospital of Stomatology, Cheeloo College of Medicine, Shandong University \& Shandong Key Laboratory of Oral Tissue Regeneration \& Shandong Engineering Laboratory for Dental Materials and Oral Tissue Regeneration, Jinan, Shandong, People's Republic of China. ${ }^{5}$ School of Medicine, Cheeloo College of Medicine, Shandong University, Jinan 250012, Shandong, China.

Received: 24 December 2020 Accepted: 7 April 2021

Published online: 20 April 2021

\section{References}

1. Camilo V, Sugiyama T, Touati E. Pathogenesis of helicobacter pylori infection. Helicobacter. 2017:22:1-6.

2. Burucoa C, Axon A. Epidemiology of helicobacter pylori infection. Helicobacter. 2017;22:1-5.

3. Al Sayed A, Anand PS, Kamath KP, Patil S, Preethanath RS, Anil S. Oral cavity as an Extragastric reservoir of helicobacter pylori. ISRN Gastroenterol. 2014; 2014:1-16. https://doi.org/10.1155/2014/261369.

4. Ansari SA, lqbal MN, Khan TA, Kazmi SU. Association of oral Helicobacter pylori with gastric complications. Life Sci. 2018;205:125-30. https://doi.org/1 0.1016/j.lfs.2018.05.026

5. Matamala-Valdés L, Sánchez-Alonzo K, Parra C, Sáez K, Aguayo-Reyes A, García A. Detection of intracellular helicobacter pylori in candida. SPP from neonate oral swabs. Rev Assoc Med Bras. 2018;64(10):928-35. https://doi. org/10.1590/1806-9282.64.10.928.

6. Kazanowska-Dygdała M, Du I, Radwan-Oczko M. The presence of helicobacter pylori in oral cavities of patients with leukoplakia and oral lichen planus. J Appl Oral Sci. 2016;24(1):18-23. https://doi.org/10.1590/1 678-775720150203.

7. Alrashdan MS, Cirillo N, McCullough M. Oral lichen planus: a literature review and update. Arch Dermatol Res. 2016;308(8):539-51. https://doi.org/1 0.1007/s00403-016-1667-2. 
8. Kurago ZB. Etiology and pathogenesis of oral lichen planus: an overview Oral Surg Oral Med Oral Pathol Oral Radiol. 2016;122(1):72-80. https://doi. org/10.1016/j.oooo.2016.03.011

9. Tampa M, Caruntu C, Mitran M, Mitran C, Sarbu I, Rusu LC, et al. Markers of oral lichen planus malignant transformation. Dis Markers. 2018;2018:7-10.

10. Sugerman PB, Savage NW, Walsh LJ, Zhao ZZ, Zhou XJ, Khan A, et al. The pathogenesis of oral lichen planus. Crit Rev Oral Biol Med. 2002;13(4):35065. https://doi.org/10.1177/154411130201300405.

11. Nosratzehi T. Oral lichen planus: an overview of potential risk factors, biomarkers and treatments. Asian Pacific J Cancer Prev. 2018;19:1161-7.

12. Attia EAS, Abdel Fattah NSA, Abdella HM. Upper gastrointestinal findings and detection of helicobacter pylori in patients with oral lichen planus: clinical dermatology • original article. Clin Exp Dermatol. 2010;35(4):355-60. https://doi.org/10.1111/j.1365-2230.2009.03464.x.

13. Hulimavu SR, Mohanty L, Tondikulam NV, Shenoy S, Jamadar S, Bhadranna A. No evidence for helicobacter pylori in oral lichen planus. J Oral Pathol Med. 2014;43(8):576-8. https://doi.org/10.1111/jop.12194.

14. Liu WZ, Xie Y, Lu H, Cheng H, Zeng ZR, Zhou LY, et al. Fifth Chinese National Consensus Report on the management of helicobacter pylori infection. Helicobacter. 2018;23:1-17.

15. Zhang Y, Wang X, Li H, Ni C, Du Z, Yan F. Human oral microbiota and its modulation for oral health. Biomed Pharmacother. 2018;99:883-93. https:// doi.org/10.1016/j.biopha.2018.01.146.

16. Kragelund $C$, Keller MK. The oral microbiome in oral lichen planus during a 1-year randomized clinical trial. Oral Dis. 2019;25(1):327-38. https://doi.org/1 0.1111/odi.12961.

17. Kamiya S, Backert S. Helicobacter pylori in Human Diseases Advances; 2018. https://doi.org/10.1007/978-3-030-21916-1.

18. Li TH, Qin Y, Sham PC, Lau KS, Chu KM, Leung WK. Alterations in gastric microbiota after H. pylori Eradication and in Different Histological Stages of Gastric Carcinogenesis. Sci Rep. 2017, 7:1-8. https://doi.org/10.1038/srep44 935.

19. Dash NR, Khoder G, Nada AM, Al Bataineh MT. Exploring the impact of helicobacter pylori on gut microbiome composition. PLoS One. 2019;14:1-13.

20. Yang L, Zhang J, Xu J, Wei X, Yang J, Liu Y, et al. Helicobacter pylori infection aggravates dysbiosis of gut microbiome in children with gastritis. Front Cell Infect Microbiol. 2019;9:1-14.

21. Yu FY, Wang QQ, Li M, Cheng YH, Cheng YSL, Zhou Y, et al. Dysbiosis of saliva microbiome in patients with oral lichen planus. BMC Microbiol. 2020; 20:1-12.

22. Wang K, Lu W, Tu Q, Ge Y, He J, Zhou Y, et al. Preliminary analysis of salivary microbiome and their potential roles in oral lichen planus. Sci Rep. 2016; 6(1):1-10. https://doi.org/10.1038/srep22943.

23. Li Y, Wang K, Zhang B, Tu Q, Yao Y, Cui B, et al. Salivary mycobiome dysbiosis and its potential impact on bacteriome shifts and host immunity in oral lichen planus. Int J Oral Sci. 2019;11(2):13. https://doi.org/10.1038/ s41368-019-0045-2.

24. Bagheri N, Azadegan-Dehkordi F, Shirzad H, Rafieian-Kopaei M, Rahimian G, Razavi A. The biological functions of IL-17 in different clinical expressions of helicobacter pylori-infection. Microb Pathog. 2015;81:33-8. https://doi.org/1 0.1016/j.micpath.2015.03.010.

25. Pekiner FN, Demirel GY, Borahan MO, Özbayrak S. Cytokine profiles in serum of patients with oral lichen planus. Cytokine. 2012;60(3):701-6. https://doi. org/10.1016/j.cyto.2012.08.007.

26. Maldonado RF, Sá-Correia I, Valvano MA. Lipopolysaccharide modification in gram-negative bacteria during chronic infection. FEMS Microbiol Rev. 2016; 40(4):480-93. https://doi.org/10.1093/femsre/fuw007.

27. Payeras MR, Cherubini K, Figueiredo MA, Salum FG. Oral lichen planus: focus on etiopathogenesis. Arch Oral Biol. 2013;58(9):1057-69. https://doi.org/10.1 016/j.archoralbio.2013.04.004.

28. Jia G, Zhi A, Lai PFH, Wang G, Xia Y, Xiong Z, et al. The oral microbiota - a mechanistic role for systemic diseases. Br Dent J. 2018;224(6):447-55. https://doi.org/10.1038/sj.bdj.2018.217.

29. de Carvalho MFMS, Cavalieri D, Do Nascimento S, Lourenço TGB, Ramos DVR. Pasqualin D da C, et al. cytokines levels and salivary microbiome play a potential role in Oral lichen Planus diagnosis. Sci Rep. 2019;9:1-10.

30. Choi YS, Kim Y, Yoon HJ, Baek KJ, Alam J, Park HK, et al. The presence of bacteria within tissue provides insights into the pathogenesis of oral lichen planus. Sci Rep. 2016;6:1-13. https://doi.org/10.1038/srep29186.

31. Arachchi PS, Fernando N, Weerasekera MM, Senevirathna B, Weerasekera DD, Gunasekara CP. Proinflammatory cytokine IL-17 shows a significant association with helicobacter pylori infection and disease severity. Gastroenterol Res Pract. 2017;2017:1-7. https://doi.org/10.1155/2017/62651 50

32. Zhou S, Huang $Y$, Liang B, Dong $H$, Yao S, Chen $Y$, et al. Systemic and mucosal pre-administration of recombinant helicobacter pylori neutrophilactivating protein prevents ovalbumin-induced allergic asthma in mice. FEMS Microbiol Lett. 2017;364:1-7.

33. Lehours P, Ferrero RL. Review: helicobacter: inflammation, immunology, and vaccines. Helicobacter. 2019;24:1-6.

34. Butz AM. Helicobacter: inflammation, immunology, and vaccines Alice. Physiol Behav. 2017;176:139-48.

35. Ikuse T, Blanchard TG, Czinn SJ. Inflammation, immunity, and vaccine development for the gastric pathogen helicobacter pylori. Curr Top Microbiol Immunol. 2019;421:1-19. https://doi.org/10.1007/978-3-030-15138$6 \_1$.

36. Jang S, Kim J, Cha JH. Cot kinase plays a critical role in helicobacter pyloriinduced IL-8 expression. J Microbiol. 2017;55(4):311-7. https://doi.org/10.1 007/s12275-017-7052-9.

37. Chua EG, Chong JY, Lamichhane B, Webberley KM, Marshall BJ, Wise MJ, et al. Gastric helicobacter pylori infection perturbs human oral microbiota. Peer J. 2019;2019:1-14

38. Lu R, Zhang J, Sun W, Du G, Zhou G. Inflammation-related cytokines in oral lichen planus: an overview. J Oral Pathol Med. 2015;44(1):1-14. https://doi. org/10.1111/jop.12142.

39. Zheng D, Liwinski T, Elinav E. Interaction between microbiota and immunity in health and disease. Cell Res. 2020;30(6):492-506. https://doi.org/10.1038/ s41422-020-0332-7.

40. Alam A, Leoni G, Quiros M, et al. The microenvironment of injured murine gut elicits a local pro-restitutive microbiota. Nat Microbiol. 2016;1:15021. https://doi.org/10.1038/nmicrobiol.2015.21

41. Baker JL, Bor B, Agnello M, Shi W, He X. Ecology of the Oral microbiome: beyond Bacteria. Trends Microbiol. 2017;25(5):362-74. https://doi.org/10.101 6/j.tim.2016.12.012.

42. Verma D, Garg PK, Dubey AK. Insights into the human oral microbiome. Arch Microbiol. 2018;200(4):525-40. https://doi.org/10.1007/s00203-018-1 505-3.

43. Schwiertz A. Netuschil NBA and L, abstract 4. Oral Microbiota. 2016;902:532. https://doi.org/10.1007/978-3-319-31248-4.

44. Murphy TF, Brauer AL, Sethi S, Kilian M, Cai X, Lesse AJ. Haemophilus haemolyticus: a human respiratory tract commensal to be distinguished from Haemophilus influenzae. J Infect Dis. 2007;195(1):81-9. https:/doi.org/10.1086/509824.

45. Mukundan D, Ecevit Z, Patel M, Marrs CF, Gilsdorf JR. Pharyngeal colonization dynamics of Haemophilus influenzae and Haemophilus haemolyticus in healthy adult carriers. J Clin Microbiol. 2007:45(10):3207-17. https://doi.org/10.1128/JCM.00492-07.

46. Kistler JO, Arirachakaran P, Poovorawan Y, Dahlén G, Wade WG. The oral microbiome in human immunodeficiency virus (HIV)-positive individuals. J Med Microbiol. 2015;64(9):1094-101. https://doi.org/10.1099/jmm.0.000128.

47. Berenson CS, Murphy TF, Wrona CT, Sethi S. Outer membrane protein p6 of nontypeable Haemophilus influenzae is a potent and selective inducer of human macrophage proinflammatory cytokines. Infect Immun. 2005;73(5): 2728-35. https://doi.org/10.1128/IAl.73.5.2728-2735.2005.

48. Essilfie AT, Simpson JL, Horvat JC, Preston JA, Dunkley ML, Foster PS, et al, Haemophilus influenzae infection drives IL-17-mediated neutrophilic allergic airways disease. PLoS Pathog. 2011;7(10):e1002244. https://doi.org/10.1371/ journal.ppat.1002244.

49. Zhang LL, Chen B, Fan XY, Wu SS, Zhang SQ, Wu HM. LPS cooperates with poly-L-arginine to promote IL-6 and IL-8 release via the JNK signaling pathway in NCl-H292 cells. J Immunol Res. 2016;2016:1-7. https://doi.org/1 $0.1155 / 2016 / 3421060$

50. Okuyama H, Tominaga A, Fukuoka S, Taguchi T, Kusumoto Y, Ono S. Spirulina lipopolysaccharides inhibit tumor growth in a toll-like receptor 4dependent manner by altering the cytokine milieu from interleukin-17/ interleukin-23 to interferon- $\gamma$. Oncol Rep. 2017;37(2):684-94. https://doi.org/1 0.3892/or.2017.5346

51. Zhao B, Li R, Yang F, Yu F, Xu N, Zhang F, et al. LPS-induced vitamin D receptor decrease in Oral keratinocytes is associated with Oral lichen Planus. Sci Rep. 2018;8:1-9.

52. Yee JKC. Are the view of helicobacter pylori colonized in the oral cavity an illusion? Exp Mol Med. 2017:49(11):e397. https://doi.org/10.1038/emm.201 7.225 . 
53. Anand PS, Kamath KP, Anil S. Role of dental plaque, saliva and periodontal disease in helicobacter pylori infection. World J Gastroenterol. 2014;20(19): 5639-53. https://doi.org/10.3748/wjg.v20.119.5639.

54. Van der Meij EH, Van der Waal I. Lack of clinicopathologic correlation in the diagnosis of oral lichen planus based on the presently available diagnostic criteria and suggestions for modifications. J Oral Pathol Med. 2003;32(9): 507-12. https://doi.org/10.1034/j.1600-0714.2003.00125.x.

55. Du GH WYF, Chen JJ, Deng YW, Han XZ, Tang GY. Potential association between Fusobacterium nucleatum enrichment on oral mucosal surface and oral lichen planus. Oral Dis. 2020;26(1):122-30. https://doi.org/10.1111/ odi.13232.

56. Wang K, Miao T, Lu W, He J, Cui B, Li J, et al. Analysis of oral microbial community and Th17-associated cytokines in saliva of patients with oral lichen planus. Microbiol Immunol. 2015;59(3):105-13. https://doi.org/1 0.1111/1348-0421.12232

57. Patel SK, Pratap CB, Jain AK, Gulati AK, Nath G. Diagnosis of helicobacter pylori: what should be the gold standard? World J Gastroenterol. 2014; 20(36):12847-59. https://doi.org/10.3748/wjg.v20.i36.12847.

\section{Publisher's Note}

Springer Nature remains neutral with regard to jurisdictional claims in published maps and institutional affiliations.

Ready to submit your research? Choose BMC and benefit from:

- fast, convenient online submission

- thorough peer review by experienced researchers in your field

- rapid publication on acceptance

- support for research data, including large and complex data types

- gold Open Access which fosters wider collaboration and increased citations

- maximum visibility for your research: over $100 \mathrm{M}$ website views per year

At BMC, research is always in progress.

Learn more biomedcentral.com/submissions 